\title{
A New Control Scheme for Improving Disturbance Rejection in Speed Control of Permanent Magnet Synchronous Motors
}

\author{
B. Srinivasan, N. J. Rao, U. R. Prasad, and A. Pittet
}

\begin{abstract}
The major hurdle encountered in achieving high performance in servo speed control is the occurrence of large relative errors at low speeds due to torque variations. In this paper, it is shown that this problem can be tackled in the case of synchronous motors, by keeping the stator current and the rotor flux nonorthogonal and including an inner position control loop to improve the transient behavior of the system. Such a scheme substantially improves disturbance rejection at the cost of accentuating the nonlinearities of the system and increasing the copper losses. In view of the pronounced nonlinearities encountered here, adaptive control using neural networks is resorted to.
\end{abstract}

\section{INTRODUCTION}

$\mathbf{H}$ IGH-performance state-of-the-art industrial servos are commonly designed using the field-oriented control technique, which consists of decoupling of the slow and fast dynamics followed by design of classical feedback controllers. This normally gives good time response to a step change in speed and reasonable rejection of torque disturbances. The change in speed caused by a torque disturbance is absolute, however, in the sense that the error is independent of the speed at which the motor is running. This means that if the operating speed range is very wide, relative errors at low speeds can be quite large. For example, with a demanded speed range of $1: 3000$ (e.g., $1-3000 \mathrm{rpm}$ ), if the error due to a change in load torque is $0.01 \%$ of the maximum speed, then the corresponding error at the minimum speed can be as high as $30 \%$. If the closed-loop system is fast enough and if the amplitude of disturbance is small, field-oriented control might be sufficient to meet the desired performance limits even with a wide speed range. In many cases, however, the system is either sluggish (due to the presence of large inertias, low sampling period, etc.) and/or the torque disturbances are large, rendering the disturbance rejection provided by a feedback controller insufficient. In short, achieving disturbance rejection becomes the bottleneck in realizing high performance, especially in applications which are characterized as above. A solution to such a problem is proposed in this paper.

Manuscript received October 2, 1994; revised July 20, 1995. Recommended by Associate Editor, B. Lohmann.

B. Srinivasan was with the Department of Computer Science and Automation, Indian Institute of Science, Bangalore-560012, India. He is now with the Institut d'Automatique, École Polytechnique Fédérale de Lausanne, CH-1015 Lausanne, Switzerland.

N. J. Rao is with the Department of Computer Science and Automation, Indian Institute of Science, Bangalore-560012, India, and the Center for Electronics Design Technology, Indian Institute of Science, Bangalore-560012, India.

U. R. Prasad is with the Department of Computer Science and Automation, Indian Institute of Science, Bangalore-560012, India.

A. Pittet is with the Center for Electronics Design Technology, Indian Institute of Science, Bangalore-560012, India.

Publisher Item Identifier S 1063-6536(96)04918-4.
In the present context, the issue of improving disturbance rejection further than what can be achieved by the best feedback controller, is addressed. Traditionally, feedforward action is used to augment the feedback loop for the rejection of disturbances [1], but is applicable only when the disturbances are available for measurement. A popular method to generate feedforward action is to use the inverse of the plant [2]. As the parameters of the plant may vary, however, the inverse should constantly keep track of the plant variations using on-line identification techniques [2]. Such a policy cannot be adopted here as most of the disturbances are either not available for measurement or expensive to measure [3]. Very little work appears to have been done for such problems. In this paper, we propose an alternative control structure for the servo speed control problem, which improves rejection of disturbances without actually having to measure them. As a result, this scheme achieves rejection of all the disturbances that arise and at all the speeds. The price paid in the scheme is having to contend with the nonlinearities of the motor, requiring the adoption of adaptive control using neural networks and the increased power dissipation. Even so, the scheme has merit since it is one of the very few methods to handle disturbance rejection effectively.

Control of electrical machines can be broadly classified into 1) closed-loop field oriented control, where the two physical quantities generating the torque (viz., the flux and the current) are kept orthogonal; and 2) open-loop control without field orientation where the two are not forced to be orthogonal [4]. $\mathrm{V} / \mathrm{f}$ control of ac machines falls under the second category. In the field-oriented control of synchronous machines, the stator current and rotor flux are kept orthogonal. As can be seen later, field orientation gives good dynamic performance but suffers from poor disturbance rejection at low speeds. This can be attributed to the fact that with a feedback structure, the change in output due to a disturbance does not depend on the present output value. In the servo control problem, this implies that the error in speed caused by a torque disturbance is independent of the speed at which the motor is running. On the other hand, we will show in the next section, that in an open-loop control structure such as the V/f control, field deorientation causes a "spring type action" internal to the motor, due to which the disturbance rejection at low speeds would be better (i.e., smaller peak deviation from set speed). The transient response is, however, in general oscillatory and inferior to the field-oriented case.

Since the disturbance rejection provided by the feedback controller is insufficient, it becomes necessary to enhance the open-loop characteristics of the system so as to meet the performance requirements. So, in this paper, we propose a 
scheme applicable to synchronous motors, which allows the stator current and the rotor flux to be nonorthogonal, so that the internal "spring effect" can be used for improving the disturbance rejection at low speeds. To achieve the desired transient response, a nonlinear position controller based on neural networks is used. The position control loop is placed inside the speed control loop to provide a more accurate "point-to-point" control of speed. Here we have analyzed and applied this control scheme to a two phase permanent magnet synchronous motor (PMSM), because of its simplicity, versatility, and growing importance in the servo systems market [5].

In the next section, we describe the new scheme for improving disturbance rejection. We also perform an approximate analysis to show that the relative error at low speeds can be improved with this scheme. Section III is devoted to the details of implementation of this scheme using neural networks. Last, conclusions are drawn in Section IV.

\section{A NEW CONTROL SCHEME FOR IMPROVING DISTURBANCE REJECTION}

\section{A. Characterization of the Inherent Feedback Loop}

Consider a two-phase permanent-magnet synchronous motor with $i_{a}$ and $i_{b}$ being the stator phase currents. The stator flux position $\rho_{s}$ and the effective stator current $I_{s}$ are given by

$$
\begin{aligned}
& \rho_{s}=\tan ^{-1}\left(\frac{i_{a}}{i_{b}}\right) \\
& I_{s}=\sqrt{i_{a}^{2}+i_{b}^{2}} .
\end{aligned}
$$

Let us assume that the current controllers are fast, and the stator flux can be positioned at its desired position. Then the torque is given by

$$
\begin{aligned}
T & =K_{T} I_{s} \sin \left(\rho_{s}-\rho_{T}\right) \\
& =K_{T} I_{s} \sin (\delta)
\end{aligned}
$$

where $K_{T}$ is the torque constant and $\delta=\left(\rho_{s}-\rho_{r}\right)$ the lag angle. It may be noted that $K_{T}$ may vary due to saturation effects. Let us assume a mechanical load with $J$ being the inertia and $B$ the friction. With $\omega_{r}$ being the rotor speed and $\omega_{\text {ref }}$ the reference speed, $T_{L}$ the lode torque, the rotor position, $\rho_{r}$, can be obtained as

$$
\rho_{r}(s)=\frac{T(s)-T_{L}(s)}{s(J s+B)} .
$$

To understand how different schemes differ in disturbance rejection characteristics, let us first study the effect of load disturbances on a motor. When the load torque increases, the speed decreases thereby decreasing $\rho_{r}$ and increasing $\delta$. In the field oriented scheme, where the controller tries to maintain $\delta=(\pi / 2)$, any increase in $\delta$, consequent to an increase in load torque, will first take it into the second quadrant, thereby reducing the generated torque, $T$. On the other hand, when the fluxes are not orthogonal i.e., $\delta<(\pi / 2), \delta$ continues to be in the first quadrant even after the load disturbance and increases the generated torque. This is an internal feedback which puts

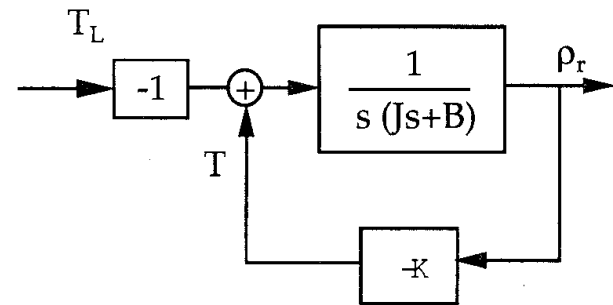

Fig. 1. Inherent loop.

the speed back to its original value without any external control action and hence contributes to the improvement of disturbance rejection.

To characterize this loop, consider a situation where the stator position $\rho_{s}$ is held constant and a torque $\Delta T_{L}$ is applied. Then, the change in rotor position, $\rho_{r}$, is given by

$$
\Delta \rho_{r}(s)=\frac{\Delta T(s)-\Delta T_{L}(s)}{s(J s+B)} .
$$

By differentiating (2), we see that the increase in the generated torque due to a change in rotor position is given, to a first-order approximation, by

$$
\begin{aligned}
\Delta T & \approx K_{T} I_{s} \cos (\delta) \Delta \delta \\
& =-K_{T} I_{s} \cos (\delta) \Delta \rho_{r} .
\end{aligned}
$$

Equations (4) and (5) are two elements of the internal loop as shown in the Fig. 1.

The closed-loop transfer function of the internal feedback loop of the motor is

$$
\begin{aligned}
\frac{\Delta \rho_{r}(s)}{\Delta T_{L}(s)} & =(-1) \frac{\frac{1}{s(J s+B)}}{1+K_{T} I_{s} \cos (\delta) \frac{1}{s(J s+B)}} \\
& =\frac{-1}{J s^{2}+B s+K_{T} I_{s} \cos (\delta)} .
\end{aligned}
$$

It can be seen that the torque generated with (5) is equivalent to having a "spring" with a spring constant $K=K_{T} I_{s} \cos (\delta)$. Examining the transfer function (6), certain remarks are in order:

Remark 1: The dc gain of the small signal system is a measure of the steady-state error in the absence of any speed controller. In the case of field oriented control for which $\delta=(\pi / 2), K=0$, and dc gain $=\infty$, the rotor angle may lag without bound. Though the system is only marginally stable (one of the characteristic root is at the origin), the other root is well damped.

Remark 2: In the case of deorientation, the dc gain is finite, as $K \neq 0$, indicating that the position lag is finite. This implies that the speed will eventually return back to the set speed without any external action. Since the characteristic roots are now complex in general, however, the response is not acceptable.

Remark 3: For large $K$, i.e., when the damping ratio $B /(2 \sqrt{J K}) \approx 0$, the change in speed will be a sinusoid with amplitude $\Delta T_{L} / \sqrt{J K}$ and natural frequency $\sqrt{K / J}$. 
Hence, the maximum change in speed due to a change in load torque is given by

$$
\left(\Delta \omega_{r}\right)_{\max }=\frac{1}{\sqrt{J K}}\left(\Delta T_{L}\right)
$$

For having the best possible rejection of disturbances i.e., low $\left(\Delta \omega_{r}\right)_{\text {max }}$, it can be seen from (7) that $K$ and hence $I_{s}$ should be as high as possible. Hence, we set $I_{s}$ to $I_{\max }$, the rated current, in the rest of the discussion.

\section{B. The Inner Position Control Loop}

The remarks of the previous section set the stage for the new scheme which has two main features: 1) allowing the stator current and the rotor flux to be nonorthogonal so as to utilize the inherent feedback loop for providing disturbance rejection and 2) using a suitable external position controller to improve the poor transient behavior caused by the inherent feedback loop.

We set $I_{s}=I_{\max }$ to make the best use of the internal feedback loop. Having thus given away one of the two degrees of freedom, we plan to improve the transient response by utilizing the other degree of freedom available in a synchronous machine i.e., the lag angle $\delta$. The control is achieved by adjusting the stator flux position $\rho_{s}$ in accordance with the variation in the rotor flux position $\rho_{r}$. In other words, a position controller is designed to meet the transient response requirements. The rotor flux position, $\rho_{r}$, can be obtained from the physical position of the rotor in synchronous machines using a position encoder. Note that the position or phase loop discussed here is not the normal position loop used in an accurate position servo. The loop under discussion is inside the speed loop for a more accurate control of speed. Let us now define the synchronous rotor flux position to be $\rho_{r s}(s)=\left[\Omega_{r e f}(s) / s\right]$. This is the position where the rotor flux should, in principle, be. So the position controller, $G_{c p}$, can then be written as

$$
\begin{aligned}
\delta & =G_{c p}\left(\rho_{r s}-\rho_{r}\right) \\
\rho_{s} & =\rho_{r}+G_{c p}\left(\rho_{r s}-\rho_{r}\right) .
\end{aligned}
$$

Note that we devised the scheme, in many respects, to be intermediate to the V/f and field-oriented control schemes and having the features of both, so that the latter may indeed be considered as special cases of the new scheme. In the present framework, $G_{c p}$ is a proportional controller of unity gain, for the V/f control scheme, i.e., $\rho_{s}=\rho_{r s}$ and the output of $G_{c p}$ is independent of the error fed to it, for field-oriented control. In one sense, the V/f scheme solely depends upon the internal feedback loop of the motor for disturbance rejection while the field-oriented control scheme completely disregards this loop. The present scheme, on the other hand, utilizes the inherent feedback mechanism of the motor for disturbance rejection and supplements it with an external one.

Since we need to improve the damping, we first look into a PD (proportional derivative) type "linear" controller, $G_{c p}(s)=K_{p}+K_{d} s$. With this setup, the closed-loop transfer function is given by

$$
\begin{aligned}
\frac{\Delta \rho_{r}(s)}{\Delta T_{L}(s)} & =\frac{-1}{J s^{2}+B s+G_{c p}(s) K} \\
& =\frac{\frac{-1}{J}}{s^{2}+\left[\frac{B+K_{d} K}{J}\right] s+\frac{K_{p} K}{J}} \\
& \propto \frac{1}{s^{2}+\frac{2}{\tau_{p m}} s+\frac{1}{\tau_{p m}^{2}}}
\end{aligned}
$$

For a critically damped response with two poles at $-1 / \tau_{p m}$, the desired closed loop transfer function is proportional to $1 /\left(\tau_{p m} s+1\right)^{2}$. By equating the actual closed-loop transfer function and the desired one, the controller gains can be obtained as

$$
\begin{aligned}
K_{p} & =\frac{J}{K \tau_{p m}^{2}} \\
K_{d} & =\frac{2 J-B \tau_{p m}}{K \tau_{p m}} .
\end{aligned}
$$

It can be seen that the dc gain and the maximum speed error due to a step change in load can be minimized by choosing a low $\tau_{p m}$. Such a "linear" scheme cannot be used directly, however, as the gains become extremely large when $\delta$ is close to $(\pi / 2),\left[\delta \rightarrow(\pi / 2) \Rightarrow K \rightarrow 0 \Rightarrow K_{p}, K_{d} \rightarrow \infty\right]$, causing instability.

\section{The Outer Speed Controller Loop}

The above problem can be tackled by choosing a proper nonlinear controller for $G_{c p}$ instead of a "linear" one. When the set speed is changed, however, the motor can only approach the new set speed with a lag due to inertia and finiteness of the torque. The controller described in (8) demands the reference and actual positions to be equal, which also means that the integrals of the set and actual speeds should match. In a bid to achieve this, the motor speed exhibits overshoots resulting in undesirable oscillatory transients. This is a standard situation when an integral type controller is used along with output saturation [2]. But, it may be realized that matching of the positions is really not required in the speed control problem and the degradation of the transient performance is rather due to the control structure used. From the definition of the synchronous rotor position we see the difference

$$
\rho_{r s}(s)-\rho_{r}(s)=\frac{1}{s}\left[\Omega_{r e f}(s)-\Omega_{r}(s)\right] .
$$

So $\rho_{r s}$ can be viewed as an integrator which integrates the speed error $\left[\omega_{r e f}-\omega_{r}\right]$ relative to the present rotor position, which causes the above mentioned problem. To solve this, the $\rho_{r s}$ integrator should not be allowed to integrate under certain conditions to avoid the oscillations arising due to nonlinearities. For this we choose

$$
\begin{aligned}
\rho_{r s}(k)= & \rho_{r s}(k-1)+\tau_{s} \omega_{r}(k) \\
& +\Theta\left[\omega_{r e f}(k)-\omega_{r}(k)\right]
\end{aligned}
$$


where $\tau_{s}$ is the sampling time, and $\Theta$ is some nonlinear function. If $\Theta=0$, there will be no integration of the error and if $\Theta=\tau_{s}\left(\omega_{r e f}-\omega_{r}\right)$, the error will be integrated always. Four possibilities exist: 1) keep $\Theta$ at one of its extremes; 2) choose a fixed value of $\Theta$ somewhere in between; 3) switch between the two extremes depending on certain switching conditions; and 4) allow $\Theta$ to be some nonlinear function of the speed error. The technique of antireset wind up [2] used with integral controllers falls under option 3), where the integration is paused whenever the output saturates. In general, the synchronous rotor position $\rho_{r s}$ can be obtained as some nonlinear combination of reference and actual speeds and the lag angle as mentioned in option 4)

$$
\begin{aligned}
\rho_{r s}(k)= & \rho_{r s}(k-1) \\
& +G_{c s}\left[\omega_{r e f}(k), \omega_{r}(k), \delta(k)\right]
\end{aligned}
$$

where $G_{c s}$ is the speed controller. Then, the position controller is given by the equation

$$
\rho_{s}(k)=\rho_{r}(k)+G_{c p}\left[\rho_{r s}(k), \rho_{r}(k)\right]
$$

Now we have some sort of a cascade controller with an inner position loop and an outer speed loop. As noted earlier, the scheme appears contrary to the normal convention, with the inner loop designed basically for a more accurate control of the speed. One may designate the inner loop a phase loop so as to conform to the normal convention, but calling it a position loop seems more appropriate.

The issues that have to be noted here are: 1) in this formulation, the problem is heavily nonlinear right from the inner loop and 2) the speed controller now faces a totally different nonlinear plant (in fact even the order of the plant is not preserved) and its task is quite different. Hence, linear techniques fail and one has to resort to nonlinear control techniques for the design of the controllers $G_{c p}$ and $G_{c s}$. In the next section, neural networks are used to this end.

In the proposed scheme, maximum current is drawn at all time instants. This will increase the copper losses inside the motor. In some situations it may be necessary to decrease the dissipation and improve the efficiency. Under such a situation, one can switch between the proposed scheme and the traditional field oriented control to get the better of both worlds. Fuzzy logic can also be used to progressively transit from de-orientation with maximum "spring effect" to a fieldoriented scheme.

\section{Approximate Analysis of the Speed Error}

Our objective in developing this scheme was to improve the relative speed error caused by a change in load torque at low speeds. We now perform an approximate analysis of the controller to find if the relative error has indeed been reduced. Let $G_{c_{\max }}$ be the maximum gain in the position loop which still keeps the lag angle $\delta$ within its saturation limits $\pm(\pi / 2)$. Even if a higher gain is incorporated in the controller, the effective gain, $G_{c_{e f f}}$, will be lower due to saturation. The value of the effective gain will be

$$
\begin{aligned}
G_{c_{e f f}} & =\frac{\frac{\pi}{2}-\delta}{\Delta \rho_{r}} \\
& \approx \frac{1}{\Delta \rho_{r}}\left(\frac{\pi}{2}-\frac{T_{L}}{K_{T} I_{\max }}\right)
\end{aligned}
$$

Equation (15) implies that $G_{c_{e f f}}$ is an increasing function of $I_{\max }$. When $K_{p}=G_{c_{e f f}}$, and $K_{d}$ is chosen appropriately, then

$$
\begin{aligned}
\frac{\left(\Delta \omega_{r}\right)_{\max }}{\Delta T_{L}} & \propto \sqrt{\frac{1}{K G_{c_{e f f}}}} \\
& \propto \frac{1}{I_{\max }}
\end{aligned}
$$

as 1) $K \propto I_{\max }$ and 2) $G_{c_{e f f}}$ increases with $I_{\max }$. If the motor is fed from a constant voltage supply of magnitude $V$, then the maximum current it can be made to draw is given by

$$
\begin{aligned}
I_{\max } & =\frac{V-K_{b} \omega_{r}}{\sqrt{R^{2}+\omega_{r}^{2} L^{2}}} \\
\approx & \frac{V}{\omega_{r} L} \\
\propto & \frac{1}{\omega_{r}} \\
\frac{\left(\Delta \omega_{r}\right)_{\max }}{\Delta T_{L}} \propto & \omega_{r} \Rightarrow \\
& \frac{\left(\Delta \omega_{r}\right)_{\max }}{\omega_{r}} \propto \Delta T_{L} .
\end{aligned}
$$

So, with this scheme, we have approximately achieved our objective of making the relative error in speed proportional to an absolute change in torque. Hence, the relative error over the desired speed range can be improved.

Remark 4: Given the same load torque, the demanded torque $\left(T_{L}+B \omega\right)$ is less at lower speeds. Hence, in the field oriented control, the current passed through the windings at low speeds is normally less than that at higher speeds. On the contrary, in the present scheme, the current drawn at lower speeds is much higher than that at higher speeds. This is deliberately intended for the sake of improving disturbance rejection. Yet only the required torque is generated by deorienting the field. The extra current, or the component of the current that does not produce a torque, gives a "reserve torque" which is called upon in the event of a disturbance.

Remark 5: At low speeds, the resistance of the windings dominates the inductance. Hence the current cannot increase with decrease in speed as given in (17). Even if a higher current is drawn, the flux produced is not increased to the desired extent due to the saturation of the core. So a relationship such as that given in (18) is quite difficult to achieve. Also, in this scheme we increase the stator copper-losses $\left(I^{2} R\right.$ losses), and the heat generated has to be properly dissipated. In short, the winding resistance, the magnetic saturation and the thermal considerations are the deciding factors which finally determine the operational limits and the level of performance at low speeds under this scheme. 


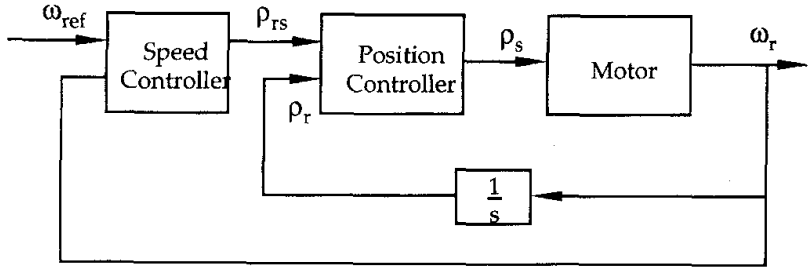

Fig. 2. Proposed scheme for servo control.

\section{IDENTIFICATION AND ADAPTIVE CONTROl USING NeURAL NETWORKS}

Now we turn towards implementing the control scheme presented in Section II. Since, the plant and the controller are both nonlinear in the present formulation, we opt for nonlinear adaptive control using neural networks for which techniques have been developed in [6]. In the indirect adaptive control scheme, the first task is the identification of the plant. As seen in Fig. 2, the plant is a mapping between the stator position $\rho_{s}$ to the rotor speed $\omega_{r}$ and rotor position $\rho_{r}$. So the identification scheme has to identify the relation between $\rho_{s}$ and $\rho_{r}$ which is a nonlinear second-order differential equation. So a neuralnetwork model used to identify this nonlinearity, with $\rho_{r_{p r e d}}$ being the predicted rotor position, is given by

$$
\rho_{r_{p r e d}}(k)=f_{i}\left[\rho_{s}(k), \rho_{r}(k-1), \rho_{r}(k-2)\right] .
$$

Since the model has no recursion, standard backpropagation can be used for learning. This system has two controllers in cascade. The inner one is the position controller whose inputs are the rotor position $\rho_{r}$ and the rotor reference position $\rho_{r s}$. The output is the desired stator position $\rho_{s}$

$$
\begin{aligned}
\rho_{s}(k) & =f_{c p}\left[\rho_{r}(k), \rho_{r}(k-1), \rho_{r s}(k), \rho_{r s}(k-1)\right] \\
I_{a}(k) & =I_{\max } \sin \left[\rho_{s}(k)\right] \\
I_{b}(k) & =I_{\max } \cos \left[\rho_{s}(k)\right] .
\end{aligned}
$$

The outer speed controller gives out the synchronous rotor position $\rho_{r s}(k)$, the inputs to which are the actual and reference speeds and stator and rotor positions. It is static and can be modeled using

$$
\begin{aligned}
\rho_{r s}(k)= & \rho_{r s}(k-1) \\
& +f_{c s}\left[\omega_{r e f}(k), \omega_{r}(k), \rho_{r}(k), \rho_{s}(k)\right] .
\end{aligned}
$$

The block diagram of the overall system is given in Fig. 3 . For the adaptation of the two controllers, the adjoint of the overall model is constructed as in Fig. 4 where the signal flow is reversed. The nodes are replaced by summing junctions and vice versa. The error is backpropagated through it for obtaining the derivatives necessary for adaptation. The adaptation is carried out using the predictive backpropagation scheme in a certainty-equivalent manner [6].

The proposed scheme was implemented on a Samarium-Cobalt PMSM. The block diagram of the experimental setup is shown in Fig. 5. The PMSM was connected to a lead screw which carried a work table. To illustrate the scenario where such a scheme is particularly useful, the parameters were so chosen that the system is sluggish and the torque

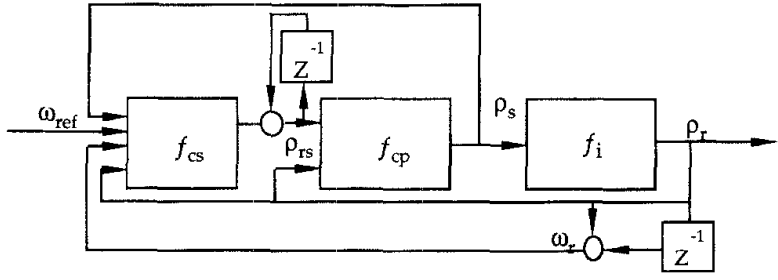

Fig. 3. Approximate model of the overall system.

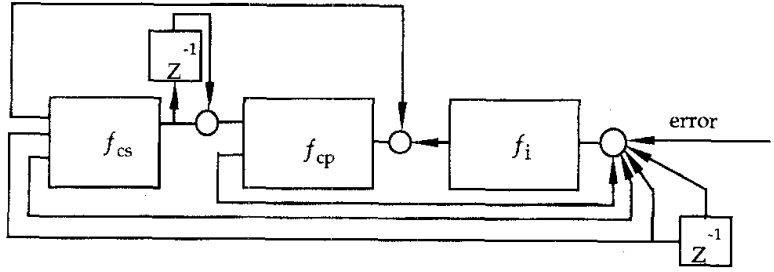

Fig. 4. Adjoint of the model.

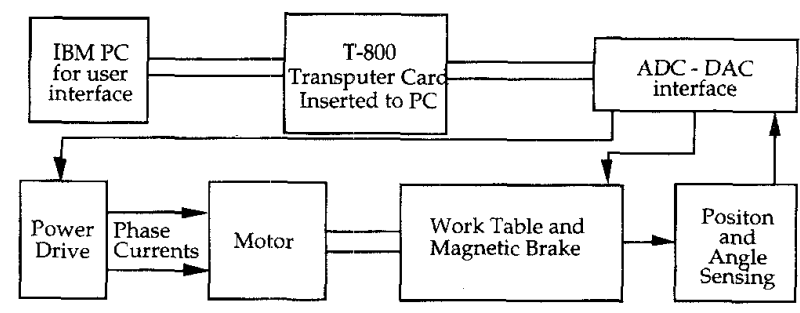

Fig. 5. Experimental setup.

variations are considerably high. The inertia was so chosen that mechanical time constant of the system was approximately $5 \mathrm{~s}$. Also, a magnetic brake was connected at one end of the shaft to generate the required torque fluctuations. The torque was varied from no load to a full load level of $10 \mathrm{Nm}$. The power drive was based on bipolar transistors. The position of rotor was obtained from an incremental rotary optical encoder connected to the shaft and the speed was obtained from the position measurement by fitting a cubic and differentiating it at the desired point. The model of the system can be described by three constants, the torque constant $K_{T}$, the reflected inertia as seen by the motor $J$ and friction $B$. The model can then be written as follows:

$$
J \ddot{\rho}_{t}(t)+B \dot{\rho}_{t}(t)=K_{T} I_{s}(t) \sin [\delta(t)]-T_{L}(t)
$$

where $\rho_{t}$ is the position of the work table, which has a oneto-one correspondence with the rotor position $\rho_{r}$, except for the backlash. Instead of obtaining an empirical model, an open loop identification of the above system was performed with the data collected, using the nonrecursive least square technique. The identification gave the parameters, $K_{T}=3.34 \mathrm{Nm} / \mathrm{Amp}$, $J=0.485 \mathrm{Kg}$, and $B=0.092 \mathrm{Kg} / \mathrm{s}$.

The neural-network algorithms, in this case, were implemented on a network of transputers programmed for the same. The controller which was a transputer card with four T-800 transputers was connected to the mother board of an IBM PC. One of the transputers handled the communication with the analog-to-digital and digital-to-analog converters and another 


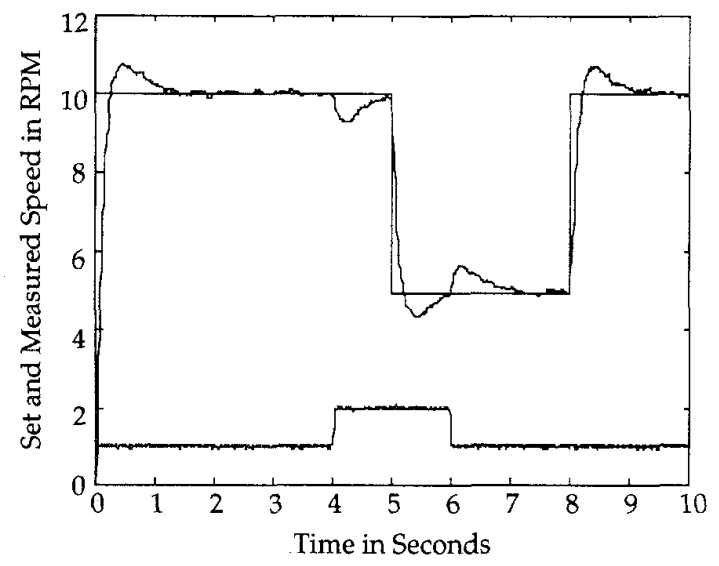

Fig. 6. Response with a traditional controller.

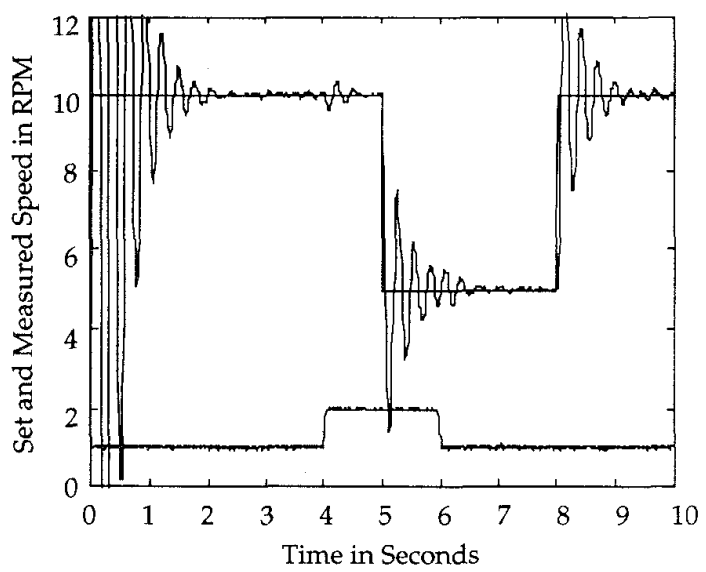

Fig. 7. Response with the new controller before learning.

handled the communication with the host PC. The control algorithm was written in OCCAM-2 and the control program was implemented in parallel between the transputers for fast computation.

The response of the system to a step load variation with a traditional field oriented controller is shown in Fig. 6. The set and the measured speeds and the load torque are plotted. The system responses before and after learning with the new control scheme are shown in Figs. 7 and 8. Oscillatory behavior is observed as expected before learning which is vastly improved after the learning is complete. The change in speed due to a $50 \%$ change in load torque is negligible

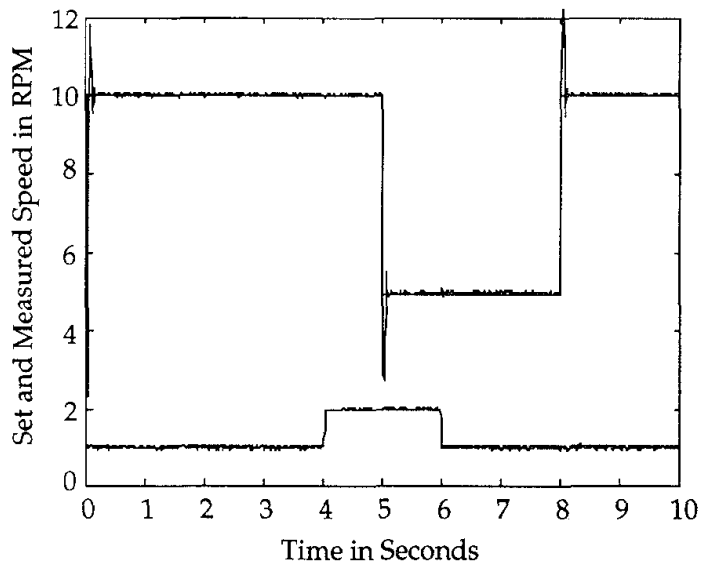

Fig. 8. Response with the new controller after learning.

and could not be detected as it was within the limits of the measurement noise.

\section{CONCLUSIONS}

In this paper, the disturbance rejection problem in the speed control of PMSM's was considered. The major bottleneck is the large relative errors caused by torque variations at low speeds. The stator current and rotor flux are deoriented to tackle this. The poor transient response resulting from this arrangement is improved by placing a position control loop inside the speed control loop. This scheme improves disturbance rejection at the cost of exposing the nonlinearities of the system and increasing the copper losses. Nonlinear adaptive control of the modified plant was performed using neural networks.

\section{REFERENCES}

[1] G. F. Franklin, J. D. Powe1l, and M. L. Workman, Digital Control of Dynamic Systems. Reading, MA: Addison-Wesley, 1990.

[2] K. J. Astrom and B. Wittenmark, Computer Controlled Systems: Theory and Design. Englewood Cliffs, NJ: Prentice-Hall, 1984.

[3] J. M. D. Murphy and F. G. Turnbull, Power Electronic Control of AC Motors. New York: Pergamon, 1988.

[4] W. Leonard, Control of Electric Drives. New York: Springer-Verlag, 1985

[5] T. Kenjo and S. Nagamori, Permanent Magnet and Brushless DC Motors. Oxford, U.K.: Oxford Univ. Press, 1985.

[6] B. Srinivasan, U. R. Prasad, and N. J. Rao, "Backpropagation through adjoints for the identification of nonlinear dynamical systems using recurrent neural networks," IEEE Trans. Neural Networks, vol. 5, pp. 213-228, Mar. 1994. 